\title{
Spatial Analysis of Low Pathogenic H5N2 Avian Influenza Outbreaks in Japan in 2005
}

\author{
Akiko NISHIGUCHI ${ }^{1)}$, Sota KOBAYASHI ${ }^{1)}$, Yoshinao OUCHI $^{2)}$, Takehisa YAMAMOTO ${ }^{1)}$, Yoko HAYAMA ${ }^{1)}$ and \\ Toshiyuki TSUTSUI ${ }^{1)}$
}

${ }^{1)}$ Epidemiology Team, National Institute of Animal Health, 3-1-5 Kannondai, Tsukuba, Ibaraki 305-0856 and

${ }^{2)}$ Department of Agriculture, Forestry and Fisheries, Ibaraki Prefecture, 978-6 Kasahara-cho, Mito, Ibaraki, Japan

(Received 29 October 2008/Accepted 14 February 2009) ABSTRACT. We conducted a spatial analysis of low pathogenic H5N2 avian influenza (AI) outbreaks, that affected 41 chicken farms in
Japan in 2005 . A statistically significant $(\mathrm{p}=0.001)$ cluster of AI-positive farms was identified in the central part of Ibaraki Prefecture.
Inside the AI cluster, the density was high for both chicken farms and chicken population, the proportion of layer finisher type farms
was high and the farm size was large. We considered it important to take precautions for AI outbreaks in densely chicken-populated
areas and to implement appropriate movement control around the affected farms to prevent transmission among farms located within
small distances in the case of AI outbreaks. Spatial scan statistics are applicable in veterinary epidemiology to detection of high risk
areas for animal diseases.

KEY WORDS: avian influenza, cluster, spatial scan statistic.

J. Vet. Med. Sci. 71(7): 979-982, 2009

On the 26th of June 2005, a H5N2 subtype of avian influenza (AI) virus was recovered from a layer chicken flock in Ibaraki Prefecture, Japan, that showed a slight decrease in egg production. Nine more virus-positive farms and 32 seropositive farms (by haemagglutination inhibition tests) were detected over the course of 26 weeks. Since the virus strain was low in pathogenicity, with an intravenous pathogenicity index [14] of 0.0 , and did not induce any particular clinical signs in the chickens of most flocks $[15,16]$, the disease spread silently and the timing of virus introduction was unclear. This strain was considered to be newly introduced into Japan, taking into account its genetic characteristics and the results of national AI monitoring implemented periodically prior to the outbreaks [9]. Of 41 affected farms, 40 were located in Ibaraki Prefecture, approximately $60 \mathrm{~km}$ north of Tokyo. In this prefecture, almost all the poultry raised were chickens; $70 \%$ of these were laying hens, and $30 \%$ were broilers.

When animal disease outbreaks occur, effective steps to explore the risk factors include investigation of the clustering of the affected farms and identification of their location and size. Nevertheless, it is generally difficult to recognize clusters visually when the locations of the cases and noncases (not affected by AI) are irregularly scattered. On the other hand, the Geographic Information System offers an opportunity to explore the associations between potential risk factors and disease incidence in combination with spatial analyses, and complements traditional visual approaches. Among the spatial analyses, spatial scan statistics enable researchers to locate statistically significant geo-

\footnotetext{
* Correspondence to: Nishiguchi, A., Epidemiology Team, National Institute of Animal, Health, 3-1-5 Kannondai, Tsukuba, Ibaraki 305-0856, Japan.

e-mail: nishiak@affrc.go.jp
}

graphical clusters (the locations of the clusters and their size) [10]. In public health epidemiology, spatial scan statistics have been applied for detecting disease clusters to enable investigation of risk factors or planning of intervention trials $[2,7,9]$. On the other hand, few spatial analyses are reported in animal diseases in Japan [13], and there are no reports concerning the clustering of animal diseases based on individual farm location. In order to investigate the risk factors in areas with a high incidence of AI, we investigated the geographical cluster of AI-case farms based on the individual farm location and their disease status using a spatial statistic, and analyzed the characteristics of the chicken farms located in the area.

The study area was defined as Ibaraki Prefecture, which is where the main outbreaks occurred. Farm status was defined according to the official report of the Ibaraki prefectural government, i.e., case farms were farms that were either virus isolation-positive or seropositive by haemagglutination inhibition tests for anti-H5 antibodies. Farms that belonged to the same owner, as well as farms located adjacently, were unified into one farm unit, and thus, there were 259 farms in total, comprising 37 case farms and 222 noncase farms. The farm profiles were obtained from a farm database managed by the prefecture's animal health administration. Farm locations were generated by converting the postal addresses of the farms into X, Y coordinates.

A spatial scan statistic was used to assess the geographical clustering of AI-case farms (AI cluster) under the Bernoulli probability model assumption [10]. This statistic uses a circular window that moves across the study area. The radius of the window varies continuously from 0 to an upper limit of not more than $50 \%$ of the total study area. The cluster assessment is performed by comparing the number of cases observed within the window with the number of 


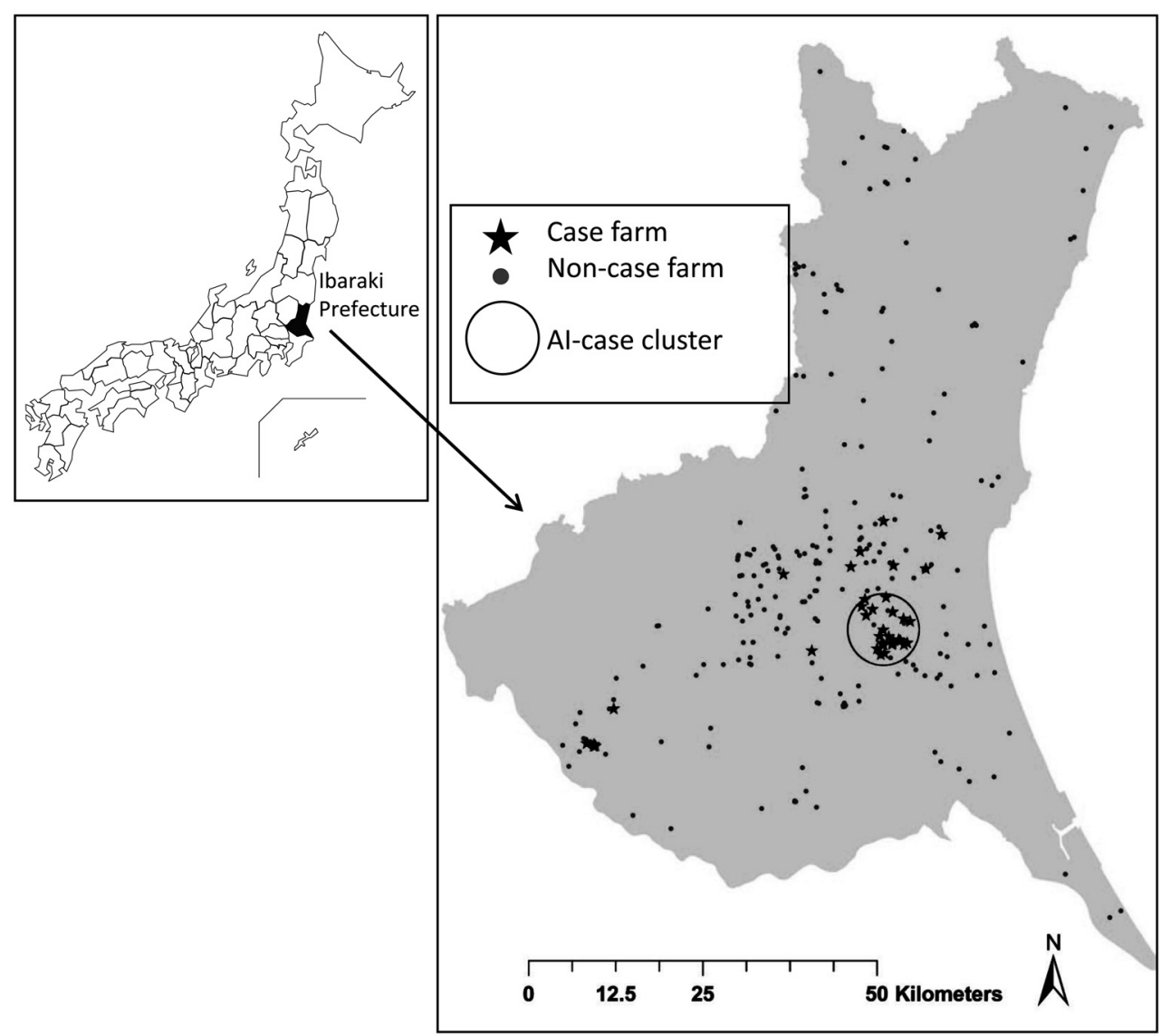

Fig. 1. Location of case / non-case chicken farms in Ibaraki prefecture, and identified AI-case cluster in H5N2 outbreaks in Japan in 2005.

expected cases in the same area on the assumption that cases are randomly distributed in space. Test of the significance of the identified cluster is based on a likelihood ratio test, the $\mathrm{p}$ value of which is obtained through Monte Carlo simulations $(p<0.05)[6]$. When the AI cluster was obtained, the production characteristics of the farms, such as farm size, use of chickens and rearing stage, were compared with the farms located inside and outside of the cluster to analyze the characteristics of farms located in high incidence areas. For statistical analyses, the Wilcoxon test was used for continuous variables, and Pearson's chi-square test was used for categorical variables. SaTScan version 7.0.1 [11] was used to detect the AI cluster, and the output was loaded into ArcGIS version 9.2 (ESRI, Redlands, CA, U.S.A.) for mapping purposes. Descriptive statistics were conducted using JMP 7 (SAS Institute Inc, Cary, NC, U.S.A.).

One significant AI cluster was detected as a circle with a radius of $5.12 \mathrm{~km}$ in the central part of Ibaraki Prefecture (Fig. 1). Sixty-five percent of the case farms (24 farms) were included inside the cluster. The ratio of AI incidence inside the cluster was 14.6 times higher than that outside the cluster ( $\mathrm{p}=0.001$, Table 1$)$.

Chicken farm density inside the cluster was approxi- mately nine times $(0.35 / 0.04)$ higher, and the density of the chicken population was approximately 32 times (40,798/ 1,287 ) higher compared with outside the cluster (Table 2). Among the various sectors, such as layers, broilers, breeders and research purpose, all farms inside the cluster belonged to the layers sector (Table 3), and the proportion of layer finisher type farms (farms with laying hens) was significantly higher inside the cluster compared with outside the cluster $(p=0.0027$, Table 3$)$. The average number of laying hens raised at one farm was approximately 100,000 inside the cluster, which was significantly larger than that outside the cluster (Table 4).

It is empirically known that AI outbreaks may become devastating in densely poultry-populated areas; the outbreaks in Italy in 1999-2003 [3-5] are one example. In this study, we showed that AI outbreaks in Japan in 2005 were clustered in areas where both chicken farm density and chicken population density were high. Larger-sized farms might have a larger amount of movement from and to the farms compared with smaller-sized farms. Once a largersized farm is affected by the AI virus, a larger amount of virus might be released, increasing the chance of the virus spreading throughout the neighboring environment. More- 
Table 1. Identified cluster of AI-case farms in H5N2 outbreaks in Japan in 2005

\begin{tabular}{ccccc}
\hline \multirow{2}{*}{$\begin{array}{c}\text { Cluster radius } \\
(\mathrm{km})\end{array}$} & \multicolumn{2}{c}{ Number of AI case farms } & & \\
\cline { 2 - 3 } & Observed & Expected & Relative risk & P value \\
\hline 5.12 & 24 & 4.14 & 14.64 & 0.001 \\
\hline
\end{tabular}

Table 2. Densities of chicken farms and chicken populations raised inside/outside of the AI-case cluster of H5N2 in Japan in 2005

\begin{tabular}{cccc}
\hline \multirow{2}{*}{ Categories } & \multirow{2}{*}{$\begin{array}{c}\text { Number } \\
\text { of } \\
\text { farms }\end{array}$} & $\begin{array}{c}\text { Density } \\
\left(\text { farms } / \mathrm{km}^{2}\right)\end{array}$ & $\begin{array}{c}\text { Chicken population } \\
\left(\text { heads } / \mathrm{km}^{2}\right)\end{array}$ \\
\hline Inside the cluster & 29 & 0.35 & 40,798 \\
Outside the cluster & 230 & 0.04 & 1,287 \\
\hline
\end{tabular}

Table 3. Comparison of farm characteristics between farms located inside and outside the AI-case cluster of H5N2 in Japan in 2005 (categorical variables)

\begin{tabular}{lcccccc}
\hline \multirow{2}{*}{ Variables } & & \multicolumn{2}{c}{ Number of farms } & & \\
\cline { 3 - 4 } Chicken use & Categories & Inside & Outside & Chi square $^{\text {a) }}$ & P value \\
\hline \multirow{5}{*}{ Existence of laying hens } & Layers & 29 & 149 & & \\
\multirow{5}{*}{ Existence of laying chicks } & Broilers & 0 & 72 & $\mathrm{NT}^{\mathrm{b})}$ & NT \\
& Breeding & 0 & 4 & & \\
& Research & 0 & 5 & & \\
& No & 24 & 123 & 9.00 & 0.0027 \\
& Yes & 5 & 107 & & \\
& No & 21 & 180 & & 0.48 \\
\hline
\end{tabular}

a) Pearson's chi square.

b) Not tested.

Table 4. Comparison of number of laying hens per farm between farms located inside and outside the AI-case cluster of H5N2 in Japan in 2005 (continuous variable)

\begin{tabular}{ccccccccc}
\hline Variables & Definition & $\mathrm{n}$ & $\mathrm{p} 10$ & $\mathrm{p} 50$ & $\mathrm{p} 90$ & Mean & SE & P value \\
\hline Number of laying & Inside & 29 & 0 & 35,000 & 300,000 & 99,495 & 171,719 & $<0.0001$ \\
hens per farm & Outside & 230 & 0 & 90 & 44,990 & 24,624 & 97,945 & \\
\hline
\end{tabular}

over, the daily practices of layer farms might increase the chance of virus introduction owing to the movement of risk materials and people, such as farm crews for egg collection, vehicles for egg shipment and egg trays, into and out of the farms. A layer finisher type farm was shown to be one of the risk factors in the AI epidemic in the Netherlands in 2003 [19].

On the other hand, tracing of epidemiological movements between farms conducted by the prefecture revealed that there were several companies whose farms were located inside the cluster, and risk materials and pepole, such as live chickens, vehicles and farm crews, had been moving between related farms before movement restriction was in place. Introduction of infected birds and contaminated fomites (egg trays, bird cages and farm equipment) or personnel are important risk factors for spreading the virus [1, $12,17]$. Therefore, it is thought that the virus was disseminated to contact farms through the movement of risk materi- als. Nevertheless, there were many farms that were not owned by any company and had no contact with other farms. Sharkey et al. [18] reported that there was no evidence of local transmission of AI virus beyond $1 \mathrm{~km}$. In our study, the average distance to the nearest farm was $750 \mathrm{~m}$, and thus, it is possible that the virus was introduced into such farms by neighborhood infection, which is characterized by transmission over a short distance via various unknown routes [19], such as via small feral mammals or birds, or unspecified movements of vehicles. Therefore, in the case of AI outbreaks, it is considered to be important to implement appropriate movement control around the affected farms to prevent transmission among farms located within small distances. In addition, it is highly recommended to take precautions for rapid detection of AI infection in densely chicken-populated areas.

In conclusion, we detected the risk factors of AI using spatial scan statistics. Although these risk factors have also 
been empirically recognized as AI risk factors in historic AI outbreaks in the world, we confirmed that spatial scan statistics are applicable in veterinary epidemiology to detection of high risk areas for animal diseases.

ACKNOWLEDGEMENTS. This study was supported by a grant-in-aid for a research project for utilizing advanced technologies in agriculture, forestry and fisheries of the Ministry of Agriculture, Forestry and Fisheries (No. 18010) of Japan.

\section{REFERENCES}

1. Alexander, D.J. 1988. Method of spread. pp. 256-272. In: Newcastle Disease (Alexander, D. J. ed.), Kluwer Academic publishers, Norwell, MA, U.S.A.

2. Bellec, S., Hemon, D., Rudant, J., Goubin, A. and Clavel, J. 2006. Spatial and space-time clustering of childhood acute leukemia in France from 1990 to 2000: a nationwide study. Br. J. Cancer 94: 763-770.

3. Busani, L., dalla Pozza, M., Bonfanti, L., Toson, M., Ferre, N. and Marangon, S. 2007. Intervention strategies for low-pathogenic avian influenza control in Italy. Avian Dis. 51: 470-473.

4. Capua, I. and Marangon, S. 2007. The challenge of controlling notifiable avian influenza by means of vaccination. Avian Dis. 51: $317-322$.

5. Capua, I., Marangon, S., dalla Pozza, M., Terregino, C. and Cattoli, G. 2003. Avian influenza in Italy 1997-2001. Avian Dis. 47: 839-843.

6. Dwass, M. 1957. Modeified randomization tests for nonparametric hypotheses. Ann. Math. Statist. 28: 181-187.

7. Gaudart, J., Poudiougou, B., Dicko, A., Rnaque, S., Toure, O., Sagara, I., Diallo, M., Diawara, S., Ouattara, A., Diakite, M. and Doumbo, O. K. 2006. Space-time clustering of childhood malaria at the household level: a dynamic cohort in Mali village. BMC Public Health 6: 286.

8. Green, C., Hoppa, R. D., Young, T. K. and Blanchard, J., F. 2003. Geographic analysis of diabetes prevalence in an urban area. Soc. Sci. Med. 57: 551-560.

9. Investigation team on the route of infection of highly pathogenic avian influenza. 2006. In: Report of the Investigation Team on the Route of infection of Highly Pathogenic Avian Influenza, Route of Infection of the Highly Pathogenic Avian
Influenza in 2005. Available by http://www.maff.go.jp/j/ syouan/douei/tori/pdf/report2005.pdf (accessed on 28 October, 2008) (in Japanese).

10. Kulldorf, M. 1997. A spatial scan statistic. Commun. Statist.Theory Meth. 26: 1481-1496.

11. Kulldorf, M. 2006. In: SaTScan Version 7.0: Software for the Spatial, Temporal, and Space-time Scan Statistics, 2006. Information Management Service, Inc. Available by http:// www.satscan.org/ (accessed on 28 October, 2008).

12. Nishiguchi, A., Kobayashi, S., Yamamoto, T., Ouchi, Y., Sugizaki, T. and Tsutsui, T. 2007. Risk factors for the introduction of avian influenza virus into commercial layer chicken farms during the outbreaks caused by a low-pathogenic H5N2 virus in Japan in 2005. Zoonoses Public Health 54: 337-343.

13. Nonaka, T., Yamamoto, T., Hashimoto, S., Nishiguchi, A., Yamane, I., Kobayashi, S. and Tsutsui, T. 2005. Geographical analysis of risk factors of bovine spongiform encephlopathy. $J$. Vet. Epidemiol. 9: 15-20 (in Japanese).

14. OIE (The World Organisation for Animal Health). In: Manual of Diagnostic Tests and Vaccines for Terrestrial Animals 2008, Chapter 2.3.4. Avian influenza. Available by http:// www.oie.int/eng/normes/mmanual/2008/pdf/2.03.04_AI.pdf (accessed on 28 October 2008).

15. Okamatsu, M., Saito, T., Mase, M., Tsukamoto, K. and Yamaguchi, S. 2007. Characterization of H5N2 influenza A viruses isolated from chickens in Japan. Avian Dis. 51: 474 475.

16. Okamatsu, M., Saito, T., Yamamoto, Y., Mase, M., Tsuduku, S., Nakamura, K., Tsukamoto, K. and Yamaguchi, S. 2007. Low pathogenicity $\mathrm{H} 5 \mathrm{~N} 2$ avian influenza outbreak in Japan during the 2005-2006. Vet. Microbiol. 124: 35-46.

17. Serratosa, J., Ribó, O., Correia, S. and Pittman, M. 2007. EFSA Scientific risk assessment on animal health and welfare aspects of avian influenza (EFSA-Q-2004-075). Avian Dis. 51: 501503.

18. Sharkey, K.J., Bowers, R.G., Morgan, K.L., Robinson, S.E. and Christley, R.M. 2008. Epidemiological consequences of an incursion of highly pathogenic H5N1 avian influenza into the British poultry flock. Proc. Biol. Sci. 275: 19-28.

19. Thomas, M. E., Bouma, A., Ekker, H. M., Fonken, A. J., Stegeman, J. A. and Nielen, M. 2005. Risk factors for the introduction of high pathogenicity Avian Influenza virus into poultry farms during the epidemic in the Netherlands in 2003. Prev. Vet. Med. 69: 1-11. 